\title{
Untangling the Relationship Between Antiretroviral Therapy Use and Incident Pregnancy: A Marginal Structural Model Analysis Using Data From 47,313 HIV-Positive Women in East Africa
}

\author{
Batya Elul, PhD, MSc,* Kara K. Wools-Kaloustian, MD,† Yingfeng Wu, PhD, $\neq$ Beverly S. Musick, MS, $\S$ \\ Harriet Nuwagaba-Biribonwoha, PhD, MD, * Denis Nash, PhD, MPH, || Samuel Ayaya, MMed, $\emptyset$ \\ Elizabeth Bukusi, MBChB, MD, MPH, PhD,\# Pius Okong, PhD, MMed,** Juliana Otieno, MD, †† \\ Deo Wabwire, MD, $t$ A Andrew Kambugu, MBChB, MMed, $\S \S$ and Constantin T. Yiannoutsos, PhD\|\|
}

Background: Scale-up of triple-drug antiretroviral therapy (ART) in Africa has transformed the context of childbearing for HIV-positive women and may impact pregnancy incidence in HIV programs.

Methods: Using observational data from 47,313 HIV-positive women enrolled at $26 \mathrm{HIV}$ clinics in Kenya and Uganda between 2001 and 2009, we calculated the crude cumulative incidence of pregnancy for the pre-ART and on-ART periods. The causal effect of ART use on incident pregnancy was assessed using inverse probability weighted marginal structural models, and the relationship was further explored in multivariable Cox models.

Results: Crude cumulative pregnancy incidence at 1 year after enrollment/ART initiation was $4.0 \%$ and $3.9 \%$ during the pre-ART

Received for publication May 11, 2015; accepted January 27, 2016.

From the *Department of Epidemiology, Mailman School of Public Health, Columbia University, ICAP at Columbia University, New York, NY; $\dagger$ Division of Infectious Diseases, Indiana University School of Medicine, Indianapolis, IN; ¥ICAP at Columbia University, New York, NY; §Department of Biostatistics, Indiana University School of Medicine, Indianapolis, IN; ||Epidemiology and Biostatistics Program, CUNY School of Public Health, New York, NY; $\uparrow$ Academic Model for Prevention and Treatment of HIV (AMPATH), Eldoret, Kenya; \#Family AIDS Care and Education Services, Nairobi, Kenya; **Nsambya Hospital, Uganda Matyr's University, Kampala, Uganda; ††Nyanza General Hospital, Kisumu, Kenya; † Makerere University-Johns Hopkins University Research Collaboration, Kampala, Uganda; §§Infectious Diseases Institute, Kampala, Uganda; and \|\| Department of Biostatistics, Indiana University Fairbanks School of Public Health, Indianapolis, IN.

The IeDEA East Africa Consortium is funded by the following institutes: the National Cancer Institute, the Eunice Kennedy Shriver National Institute of Child Health \& Human Development, and the National Institute of Allergy and Infectious Diseases Grant U01AI069911-01.

The authors have no conflicts of interest to disclose.

Supplemental digital content is available for this article. Direct URL citations appear in the printed text and are provided in the HTML and PDF versions of this article on the journal's Web site (www.jaids.com).

Correspondence to: Batya Elul, PhD, Department of Epidemiology, Mailman School of Public Health, Columbia University, 722 West 168th Street, Room 528, New York, NY 10032 (e-mail: be2124@columbia.edu).

Copyright $\mathbb{C} 2016$ Wolters Kluwer Health, Inc. All rights reserved. This is an open access article distributed under the terms of the Creative Commons Attribution-NonCommercial-NoDerivatives License 4.0 (CC BY-NC-ND), which permits downloading and sharing the work provided it is properly cited. The work cannot be changed in any way or used commercially. and on-ART periods, respectively. In marginal structural models, ART use was not significantly associated with incident pregnancy [hazard ratio $=1.06 ; 95 \%$ confidence interval $(\mathrm{CI}): 0.99$ to 1.12 ] Similarly, in Cox models, there was no significant relationship between ART use and incident pregnancy (cause-specific hazard ratio: $0.98 ; 95 \% \mathrm{CI}: 0.91$ to 1.05$)$, but effect modification was observed. Specifically, women who were pregnant at enrollment and on ART had an increased risk of incident pregnancy compared to those not pregnant at enrollment and not on ART (cause-specific hazard ratio: $1.11 ; 95 \% \mathrm{CI}: 1.01$ to 1.23 ).

Conclusions: In this large cohort, ART initiation was not associated with incident pregnancy in the general population of women enrolling in HIV care but rather only among those pregnant at enrollment. This finding further highlights the importance of scaling up access to lifelong treatment for pregnant women.

Key Words: pregnancy incidence, ART, HIV/AIDS, sub-Saharan Africa

(J Acquir Immune Defic Syndr 2016;72:324-332)

\section{INTRODUCTION}

The massive expansion of HIV prevention, care, and treatment services in sub-Saharan Africa over the past decade has transformed the context of childbearing for women living with HIV in the region. HIV-positive women are living longer, healthier, and more productive lives ${ }^{1-3}$ and, for the first time since the start of the epidemic, face a real opportunity of bearing HIV-negative children., ${ }^{4,5}$ Whether and how these new realities impact pregnancy incidence among women enrolled in HIV programs have important implications for the organization of services and the clinical management of women's health.

Although numerous qualitative and quantitative studies suggest that women on antiretroviral therapy (ART) are reconsidering their pregnancy desires in favor of childbearing, ${ }^{6-9}$ far less is known about the relationship between ART initiation and incident pregnancy. To date, only 2 studies have directly examined whether ART initiation alters pregnancy incidence. ${ }^{6,10}$ Both found that women on ART were more 
likely to experience incident pregnancy than women in preART care but suffered from limited generalizability - the first comprised women who were pregnant at enrollment in HIV care or recently postpartum, and enrolled through prevention of mother-to-child transmission (PMTCT) programs, and the second was limited to a single site in a large African city. Additionally, although these studies provided insights into patient-level factors associated with incident pregnancy, information is needed on whether site-level factors (eg, availability of PMTCT services, location of family planning services) are associated with incident pregnancy.

We used data from a large sample of women of reproductive age receiving HIV services in diverse settings in Kenya and Uganda to estimate the potential influence of ART initiation on the incidence of pregnancy. In addition to offering more generalizable results than previous studies and examining the potential relationship between site-level factors and incident pregnancy, the methods used in this article account for 3 critical factors that directly affect estimation of pregnancy incidence: (1) the propensity to initiate ART, and the resulting longitudinal confounding when examining the causal relationship between ART use and incident pregnancy; (2) prevalent pregnancy (ie, pregnancy at enrollment in HIV care) as a factor associated both with the likelihood of enrollment in HIV care and the likelihood of ART initiation; and (3) the competing events of death and loss to program, both of which occur frequently among patients newly enrolling in care.

\section{METHODS}

\section{Study Design and Study Population}

We conducted an observational cohort analysis using patient- and site-level data from the East African regional consortium of the International Epidemiologic Databases to Evaluate AIDS collaboration (IeDEA; www.iedea.org). Patient information routinely collected during clinic visits was documented by clinicians on national or clinic-developed clinical encounter forms, entered into on-site databases by data clerks, and transmitted to the IeDEA East African Regional Data Center. We evaluated data from women aged 15 to 49 years not known to have been sterilized or to have had a hysterectomy, who enrolled in HIV care between November 27, 2001 and May 8, 2009, and who made at least 1 clinic visit after enrollment or, if pregnant at enrollment, after delivery. The exact date of database closure differed by site and ranged from March 2008 to May 2009. Site-level data were obtained through a closed-ended facility survey completed by facility staff in late 2009 and were assumed to apply to the entire study period. ${ }^{11}$ The survey documented the context in which facilities operate and specific services available at each facility. Use of these data for research purposes was approved by institutional review bodies at participating clinics and universities.

\section{Data Management}

The study outcome, first incident pregnancy, was defined as the first pregnancy observed for women who were not pregnant at enrollment in HIV care or the first subsequent pregnancy observed for women who were pregnant at enrollment. Conception dates were used to determine if the incident pregnancy occurred in the pre-ART or on-ART period and were estimated using several pregnancy-related measures available in the databases, including: (1) estimated gestational age at clinic visit (based on physical examination); (2) date of last menstrual period (LMP); (3) estimated or actual date of delivery; and (4) pregnancy status at each clinic visit. When a gestational age was available, the date of conception was calculated by backdating the date at which the pregnancy was detected by the recorded gestational age. When no gestational age was available but a date of delivery was recorded, the pregnancy was assumed to have been carried to term and conception was estimated as occurring 280 days earlier. When neither a gestational age nor a date of delivery was available but a date of LMP was recorded, conception was calculated as occurring 14 days after the LMP. Finally, when only the woman's pregnancy status was recorded at 1 or more visits, we assumed a median gestational age of 147 days at first detection and backdated the date of conception accordingly. The median gestational age of 147 days was based on the gestational age at pregnancy identification among women in facilities that recorded gestational age and/or LMP.

Our primary exposure of interest was therapeutic ART use. ART was defined as triple therapy and was treated as a time-dependent variable in all analyses to account for different levels of risk before and after ART initiation for any given patient. Women were considered eligible for ART based on the criteria that were in place at the time of treatment initiation. Women who initiated triple-therapy ART for PMTCT, discontinued it after delivery and later became pregnant again were classified as not on ART at the time of conception. Body weight, World Health Organization (WHO) stage, and CD4 count were among the other patient-level variables of interest in our analyses and subject to missing values. Missing weight, WHO stage, and CD4 count across all visits were imputed by linear interpolation (when 2 values were available) or by carrying forward the last available value (when interpolation was impossible). After these procedures, the proportion of women missing weight, WHO stage, and CD4 count was $<2 \%$.

\section{Analysis}

Pregnancy-free survival was calculated from enrollment in HIV care (for women who were not pregnant at enrollment) or from the estimated or recorded date of delivery (for women who were pregnant at enrollment in HIV care) until: (1) the time of conception of the first incident pregnancy or (2) the time of the last clinic visit recorded before database closure. Censoring was a result of transfer, death, or loss to follow-up (LTFU); the latter was defined as patients without evidence of transfer or death and with no visits for at least 12 months before database closure for pre-ART patients and 6 months for patients on ART. The crude cumulative incidence of pregnancy and $95 \%$ confidence intervals (CIs) were calculated using the nonparametric estimation method of Aalen and 
Johansen, ${ }^{12}$ with death and LTFU considered as competing events. This approach is preferable to using the complement of the Kaplan-Meier survival estimate or any other model that considers death and LTFU as censoring events because such estimates are upwardly biased; indeed, they do not allow deaths before pregnancy and consequently anticipate additional pregnancies in the death and LTFU groups. ${ }^{13,14}$ The pre-ART cumulative incidence of pregnancy was calculated censoring data at the time of ART initiation. The on-ART cumulative incidence of pregnancy was estimated considering women "at risk" for an incident pregnancy only during the time since ART initiation (left-truncated data).

We assessed the causal effect of ART on pregnancy using stabilized inverse probability weighted marginal structural models that adjusted for potential longitudinal confounding because of initiation of ART. In other words, these models account for the fact that ART may be initiated differentially for various patient subgroups over time, and the very membership in these subgroups may be associated with the likelihood of incident pregnancy. A generalized estimating equation model with a logit link was used to generate the estimated probability of starting ART based on a number of patient-level predictive factors recorded at enrollment and over time, including CD4 count, WHO stage, body weight, pregnancy status, the time of each clinic visit relative to enrollment (expressed as both a linear and a quadratic trend), and the HIV program where the patient was receiving services [the Academic Model Providing Access to Healthcare (AMPATH), www.ampathkenya.org; the MTCT-Plus Initiative, www.icap.columbia.edu; the Infectious Diseases Institute, and Family AIDS Care and Education Services (FACES) Partnership, www.faces-kenya.org]. To examine the robustness of our findings, we compared results from the marginal structural model including all patients to a model that only included women who were pregnant at enrollment in care. Stabilized inverse probability weights were determined following the methods of Cole and Hernán (see Tables S1 and S2, Supplemental Digital Content, http://links.lww.com/QAI/A795). ${ }^{15}$

As a secondary approach, we conducted an unweighted analysis using unadjusted and multivariable Cox proportional hazard models. Although these are not causal models in the strict sense, we include them to allow "backward compatibility" with previous publications. ${ }^{6,10}$ Patient-level measures of interest included age and pregnancy status at enrollment, enrollment year, and WHO stage, and CD4 count recorded at enrollment and over time. Regarding site-level factors, we included program model, facility location (rural, semi-urban, urban), facility type (teaching/referral hospitals, district hospitals/sub-district hospitals, rural health centers), location of PMTCT services (integrated within the HIV clinic, elsewhere in facility, at another facility), and location of family planning services (integrated within the HIV clinic, elsewhere in the facility). For the multivariable analysis, factors significant at $\alpha=0.20$ level in unadjusted analyses were added to a model that included ART using a forward stepwise approach and were evaluated for their effect on the risk of incident pregnancy and their confounding effect with ART initiation. We also included an interaction term to determine whether the relationship of ART and incident pregnancy was modified by pregnancy status at enrollment in care. Factors significant at $\alpha=0.05$ level were retained in the multivariable model. In the context of the competing risk framework, this model, which censors death and LTFU, estimates causespecific hazard rates (CSHR). ${ }^{16}$

Analyses were conducted using SAS 9.2 (SAS Institute, Cary, NC). Interpolation and imputation of missing data were performed with STATA version 13.0 (Stata Corp, College Station, TX).

\section{RESULTS}

\section{Patient and Site Characteristics}

Overall, 47,313 women enrolled in HIV care during the study period and met the study eligibility criteria, $10 \%$ of whom were pregnant at enrollment (Table 1). Most $(86 \%)$ were enrolled between 2005 and 2009. At enrollment, women averaged 32.5 years and about one-third were known to be married or living with a partner. Only $4 \%$ of women were known to be nulliparous at enrollment, and among those with information on prior births, median parity was 3. Approximately one-third of patients were enrolled with a WHO stage of III/IV and the median CD4 count at enrollment (assessed among $83 \%$ of women) was 230 cells per microliter (interquartile range: 99-418). Sixty-one percent of women had initiated ART by the end of the observation period, such that $39 \%$ of the women were observed only during the preART period, $8 \%$ were observed only on ART, and $53 \%$ were observed both before and after ART initiation.

Table 2 shows site-level characteristics of included facilities. Women received care in 26 facilities: 23 in Kenya and 3 in Uganda. Eighteen facilities were part of the AMPATH Consortium, 3 were affiliated with the MTCTPlus Initiative, 4 were from the FACES Partnership, and the remaining site was not affiliated with a larger program model. Half of the facilities were located in urban areas. Thirteen offered family planning services within the HIV care clinic. PMTCT services were integrated in the HIV clinic in 13 facilities.

\section{Crude Cumulative Incidence of Pregnancy}

The cumulative incidence of pregnancy at 1 year from enrollment was $7.0 \%$. The incidence 1 year after enrollment for those in pre-ART care was $4.0 \%$ and 1 year after ART initiation for those on ART was 3.9\% (Table 3). The cumulative incidence of pregnancy for both the pre-ART and the on-ART periods was highest for women aged 20 years or younger (pre-ART: $8.7 \%, 95 \% \mathrm{CI}$ : 6.8 to 11.0 ; on ART: $9.7 \%$, 95\% CI: 6.5 to 14.4 ) and those who were married or living with a partner (pre-ART: $7.4 \%, 95 \% \mathrm{CI}$ : $7.0 \%$ to $7.9 \%$; on ART: $5.3 \%, 95 \% \mathrm{CI}: 4.8 \%$ to $6.0 \%$ ) and had baseline CD4 counts $\geq 350$ cells per microliter (preART: $9.1 \%, 95 \%$ CI: $8.6 \%$ to $9.7 \%$; on ART: $6.0 \%, 95 \%$ CI: $4.9 \%$ to $7.2 \%$ ). Additionally, the cumulative incidence of pregnancy was higher at 1 year in the pre-ART period 
TABLE 1. Sociodemographic and Clinical Characteristics of Study Participants*

\begin{tabular}{|c|c|}
\hline & n (\%) \\
\hline No. participants & $47,313(100.0)$ \\
\hline \multicolumn{2}{|l|}{ Age at enrollment, yr } \\
\hline Median, IQR & $32.5(27.6-38.6)$ \\
\hline \multicolumn{2}{|l|}{ Enrollment year } \\
\hline 2001-2004 & $6403(13.5)$ \\
\hline $2005-2006$ & $20,997(44.4)$ \\
\hline $2007-2009$ & $19,913(42.1)$ \\
\hline \multicolumn{2}{|l|}{ Civil status } \\
\hline Never married/not living with partner & $3749(7.9)$ \\
\hline Married/living with partner & $15,187(32.1)$ \\
\hline Divorced/separated & $5292(11.2)$ \\
\hline Widowed & $8381(17.7)$ \\
\hline Missing & $14,704(31.1)$ \\
\hline \multicolumn{2}{|l|}{ Number of live births } \\
\hline Median, IQR & $3.0(2.0-5.0)$ \\
\hline 0 & $1698(3.6)$ \\
\hline $1-2$ & $12,724(26.9)$ \\
\hline $3-4$ & $11,277(23.8)$ \\
\hline$\geq 5$ & $9242(19.5)$ \\
\hline Missing & $12,372(26.2)$ \\
\hline \multicolumn{2}{|l|}{ Pregnant at enrollment } \\
\hline Yes & $4487(9.5)$ \\
\hline No & $42,826(90.5)$ \\
\hline \multicolumn{2}{|l|}{ Weight at enrollment, $\mathrm{kg}$} \\
\hline Median (IQR) & $54.0(47.5-61.0)$ \\
\hline \multicolumn{2}{|l|}{ WHO stage at enrollment } \\
\hline $\mathrm{I} / \mathrm{II}$ & $26,278(55.5)$ \\
\hline III/IV & $16,330(34.5)$ \\
\hline Missing & $4705(9.9)$ \\
\hline \multicolumn{2}{|l|}{ CD4 cells per microliter at enrollment } \\
\hline Median (IQR) & $230.0(99-418)$ \\
\hline $0-99$ & $9789(20.7)$ \\
\hline $100-199$ & $7812(16.5)$ \\
\hline $200-349$ & $8816(18.6)$ \\
\hline$\geq 350$ & $12,713(26.9)$ \\
\hline Missing & $8183(17.3)$ \\
\hline \multicolumn{2}{|l|}{ Initiated ART during study period } \\
\hline Yes & $28,652(60.6)$ \\
\hline No & $18,661(39.4)$ \\
\hline
\end{tabular}

than in the on-ART period for all subgroups examined, with the exception of the following: nulliparous women, women with low CD4 counts at enrollment $(\leq 199$ cells $/ \mu \mathrm{L})$, and women enrolled at facilities that referred women off-site for PMTCT services.

\section{Factors Associated With Incident Pregnancy}

Table 4 shows results from the: (1) marginal structural model weighted for the propensity of ART initiation; (2) unadjusted Cox models; (3) adjusted Cox model; (4) adjusted Cox model with the addition of the interaction term for pregnancy status at enrollment and ART status. The
TABLE 2. Site Characteristics

\begin{tabular}{lc}
\hline & $\mathbf{n}(\mathbf{\%})$ \\
\hline Number of sites & $26(100.0)$ \\
Country & $23(88.0)$ \\
Kenya & $3(12.0)$ \\
Uganda & \\
Program & $18(69.2)$ \\
AMPATH & $3(11.5)$ \\
MTCT-plus & $1(3.9)$ \\
Infectious Diseases Institute & $4(15.4)$ \\
FACES & \\
Facility location & $13(50.0)$ \\
Urban & $7(26.9)$ \\
Semi-urban & $6(23.1)$ \\
Rural & \\
Facility type & $4(15.4)$ \\
Referral/teaching hospital & $12(46.2)$ \\
District hospital & $10(38.5)$ \\
Health center & \\
Availability of family planning within HIV clinic & $13(50.0)$ \\
Yes & $13(50.0)$ \\
No & \\
Availability of PMTCT within facility & $13(50.0)$ \\
Embedded in HIV clinic & $10(38.5)$ \\
Elsewhere in facility & $3(11.5)$ \\
Off-site &
\end{tabular}

marginal structural model showed a nonsignificant association between ART use and incident pregnancy (adjusted hazard ration [aHR]: $1.06,95 \% \mathrm{CI}$ : 0.99 to 1.12 ) (column 1). ART use was associated with an $11 \%$ lower hazard of incident pregnancy in the unadjusted Cox model (column 2), but no difference was observed after adjustment for other patient-level and site-level variables (CSHR: 0.98, 95\% CI: 0.91 to 1.05 ) (column 3 ). The addition of an interaction term between pregnancy status at enrollment in HIV care and time-dependent ART status (column 4) showed that the relationship between ART use and incident pregnancy was modified by pregnancy status at enrollment. Relative to women who were not pregnant at enrollment and not on ART, no significant difference was observed in the hazard of pregnancy among women who were not pregnant at enrollment and on ART, whereas women who were pregnant at enrollment and not on ART were significantly less likely to have an incident pregnancy (adjusted cause-specific hazard ratio [aCSHR]: $0.78,95 \% \mathrm{CI}$ : 0.61 to 0.98 ). ART use among women who enrolled when pregnant was associated with an 11\% higher hazard of a subsequent pregnancy compared to women who were not pregnant when enrolled and not on ART (aCSHR: 1.11, 95\% CI: 1.01 to 1.23). The sensitivity analysis that used a marginal structural model to examine the relationship between ART use and incident pregnant only among women who were pregnant at enrollment yielded a hazard ratio $=1.31(95 \%$ CI: 0.92 to 1.86 ).

Regarding other patient-level factors independently associated with incident pregnancy in the adjusted Cox model 
TABLE 3. Crude Cumulative Incidence of Pregnancy (and 95\% Cls) 1 Year After Enrollment in HIV Care (All Patients), 1 Year After Enrollment in HIV Care (Pre-ART Patients Only), and 1 Year After ART Initiation (ART Patients Only)

\begin{tabular}{|c|c|c|c|}
\hline & \multicolumn{3}{|c|}{ Cumulative Incidence $(95 \% \mathrm{CI}) *$} \\
\hline & Overall & Pre-ART Period & On-ART Period \\
\hline Overall & 7.9 (7.6 to 8.2$)$ & $4.0(3.8$ to 4.2$)$ & $3.9(1.5$ to 4.2$)$ \\
\hline \multicolumn{4}{|l|}{ Age at enrollment, yr } \\
\hline$<20$ & $14.2(11.6$ to 17.3$)$ & $8.7(6.8$ to 11.0$)$ & $9.7(6.5$ to 14.4$)$ \\
\hline $20-29$ & 13.5 (12.9 to 14.2$)$ & $9.0(8.5$ to 9.5$)$ & $7.0(6.3$ to 7.7$)$ \\
\hline \multicolumn{4}{|l|}{ Enrollment year } \\
\hline 2001-2004 & $5.4(4.8$ to 6.0$)$ & $3.8(3.4$ to 4.4$)$ & $2.5(1.9$ to 3.1$)$ \\
\hline $2005-2006$ & $8.4(8.0$ to 8.8$)$ & $5.4(5.0$ to 5.7$)$ & $4.4(4.0$ to 4.9$)$ \\
\hline 2007-2009 & $8.2(7.7$ to 8.7$)$ & $5.5(5.1$ to 5.9$)$ & $3.6(3.1$ to 4.1$)$ \\
\hline \multicolumn{4}{|l|}{ Education level } \\
\hline None & 5.4 (3.9 to 7.4$)$ & 3.9 (2.6 to 5.6$)$ & 2.4 (1.2 to 4.7$)$ \\
\hline Never married/not living with partner & $6.7(5.7$ to 7.9$)$ & 4.7 (4.0 to 5.6$)$ & $2.7(1.9$ to 3.9$)$ \\
\hline Married/living with partner & $11.5(10.9$ to 12.1$)$ & $7.4(7.0$ to 7.9$)$ & $5.3(4.8$ to 6.0$)$ \\
\hline Divorced/separated & $6.7(5.9$ to 7.6$)$ & $4.1(3.5$ to 4.7$)$ & $3.6(2.8$ to 4.5$)$ \\
\hline Widowed & $4.2(3.7$ to 4.7$)$ & $2.8(2.4$ to 3.3$)$ & $2.0(1.6$ to 2.6$)$ \\
\hline \multicolumn{4}{|l|}{ Number of live births } \\
\hline 0 & $10.1(8.5$ to 12.1$)$ & $5.1(4.0$ to 6.4$)$ & $6.1(4.5$ to 8.3$)$ \\
\hline $1-2$ & $9.9(9.3$ to 10.6$)$ & $6.3(5.8$ to 6.8$)$ & $4.8(4.3$ to 5.5$)$ \\
\hline $3-4$ & $9.3(8.6$ to 9.9$)$ & $6.0(5.5$ to 6.5$)$ & $4.5(3.9$ to 5.2$)$ \\
\hline$\geq 5$ & $5.8(5.2$ to 6.3$)$ & $4.1(3.7$ to 4.6$)$ & $2.6(2.1$ to 3.2$)$ \\
\hline \multicolumn{4}{|l|}{ Pregnant at enrollment } \\
\hline Yes & $14.1(12.9$ to 15.3$)$ & $4.6(4.0$ to 5.2$)$ & $6.6(5.8$ to 7.6$)$ \\
\hline \multicolumn{4}{|l|}{ CD4 cells per microliter at enrollment } \\
\hline \multicolumn{4}{|l|}{ Program } \\
\hline AMPATH & $8.4(8.1$ to 8.8$)$ & $5.5(5.2$ to 5.7$)$ & $4.2(3.8$ to 4.5$)$ \\
\hline MTCT-plus & $9.7(8.1$ to 11.6$)$ & $7.5(6.1$ to 9.1$)$ & $2.7(2.2$ to 3.4$)$ \\
\hline Infectious Diseases Institute & $5.1(4.6$ to 5.8$)$ & $3.2(2.7$ to 3.7$)$ & $3.2(1.8$ to 5.4$)$ \\
\hline FACES & $7.0(6.2$ to 8.0$)$ & $5.3(4.7$ to 6.2$)$ & $3.8(2.7$ to 5.4$)$ \\
\hline \multicolumn{4}{|l|}{ Facility location } \\
\hline Urban & $8.7(7.9$ to 9.5$)$ & $5.8(5.2$ to 6.5$)$ & $4.4(3.6$ to 5.3$)$ \\
\hline Semi-urban & $8.1(7.6$ to 8.7$)$ & $5.3(4.9$ to 5.8$)$ & $4.0(3.5$ to 4.6$)$ \\
\hline Rural & $8.2(7.8$ to 8.7$)$ & $5.4(5.0$ to 5.7$)$ & $4.2(3.7$ to 4.7$)$ \\
\hline \multicolumn{4}{|l|}{ Facility type } \\
\hline Referral/teaching hospital & $7.0(6.6$ to 7.5$)$ & $4.6(4.3$ to 5.0$)$ & $3.3(2.9$ to 3.7$)$ \\
\hline District hospital & $9.3(8.7$ to 9.9$)$ & $6.1(5.7$ to 6.6$)$ & $4.4(3.9$ to 5.0$)$ \\
\hline Health center & $7.5(7.1$ to 8.0$)$ & $5.0(4.6$ to 5.3$)$ & $4.1(3.6$ to 4.7$)$ \\
\hline \multicolumn{4}{|l|}{ Availability of PMTCT within facility } \\
\hline Embedded in HIV clinic & $8.4(7.9$ to 8.9$)$ & $5.6(5.2$ to 6.0$)$ & $4.2(3.7$ to 4.7$)$ \\
\hline Elsewhere in facility & $8.2(7.7$ to 8.7$)$ & $5.3(5.0$ to 5.7$)$ & $4.1(3.7$ to 4.6$)$ \\
\hline Off-site & $6.6(4.2$ to 0.3$)$ & $4.1(2.1$ to 6.9$)$ & $4.8(2.3$ to 9.8$)$ \\
\hline
\end{tabular}

*At 12 months after enrollment (pre-ART period) or 12 months after ART initiation (among women with no previous incident pregnancies who started ART). 
TABLE 4. Inverse Probability Weighted, Unadjusted, and Adjusted Hazard of Incident Pregnancy ( $\mathrm{n}=47,313$ Women)

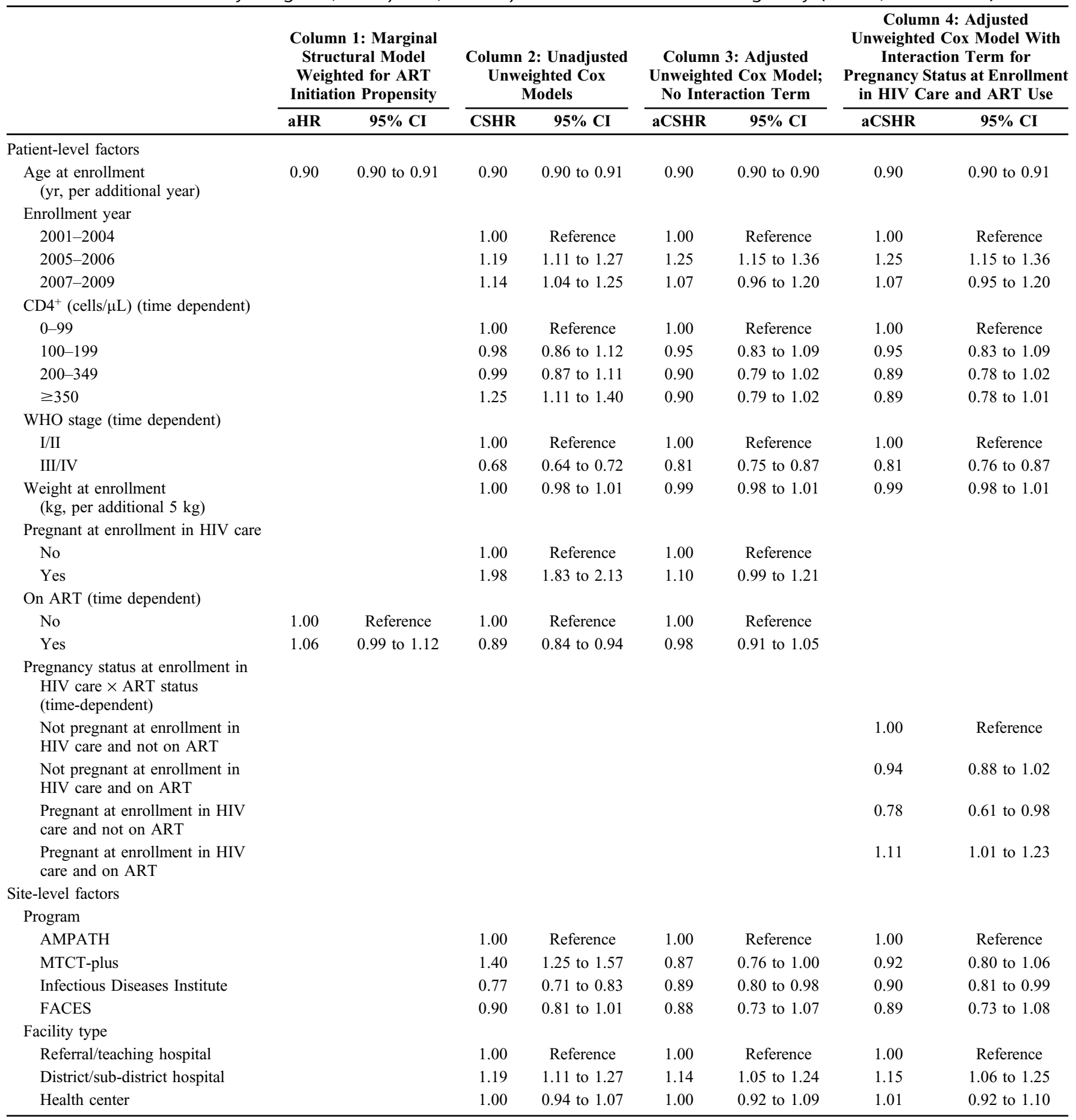

(column 4), patients enrolling in HIV care in 2005-2006 relative to the very early phase of HIV service scale-up (2001-2004) were more likely to have an incident pregnancy, whereas those with WHO Stage III/IV vs. I/II were less likely to experience an incident pregnancy. With respect to site-level factors, relative to women enrolled at AMPATH, those enrolled at the Infectious Diseases Institute had a decreased risk of incident pregnancy, whereas those in the MTCT-Plus Initiative, most of whom were pregnant at enrollment, had a significantly higher risk of incident pregnancy. In addition, women enrolled at district/subdistrict hospitals had an increased risk of incident pregnancy compared to those 
enrolled at tertiary-level facilities. On-site availability of PMTCT services and integration of family planning services in the HIV care clinic were not significantly associated with incident pregnancy in unadjusted or adjusted analyses.

\section{DISCUSSION}

In this study, conducted in its entirety before the roll out of PMTCT option B+, we examined data from a cohort of 47,313 HIV-positive women at $26 \mathrm{HIV}$ clinics in Kenya and Uganda and found that the relationship between ART initiation and incident pregnancy is nuanced and substantially weaker than previously reported. Whereas the 2 previous studies that included patients both in pre-ART care and on-ART reported an increased incidence of pregnancy following ART initiation, ${ }^{6,10}$ in our study, this relationship was not observed when we conducted a more rigorous causal analysis using marginal structural models and observed only among the subset of women who were pregnant at enrollment in HIV care in our secondary analysis that used similar methods to those used in the previous studies. Of note, one of these earlier studies comprised women who were pregnant or recently postpartum at enrollment in HIV care ${ }^{10}$ whereas the other did not examine the potential effect modification by baseline pregnancy status. $^{6}$ One possible explanation for an increased likelihood of incident pregnancy while on ART among women who were pregnant at enrollment in HIV care is that pregnancies at enrollment represent a large number of factors that predispose women to both ART initiation (as pregnant women are more likely to be identified by and subsequently enroll in HIV programs) and incident pregnancy (as women who are pregnant at enrollment have demonstrated the ability, desire, and/or availability of the proper environment to get pregnant). Analyses conducted to generate the weights for the marginal structural models show that women who were pregnant at enrollment were substantially more likely to be initiated on ART than women who were not pregnant at enrollment (see Tables S1 and S2, Supplemental Digital Content, http://links.lww.com/QAI/A795). Additionally, women who were pregnant at enrollment in HIV care may have received PMTCT services during their previous pregnancy and thus have increased knowledge of the prevention benefits of ART, which in turn may have affected their subsequent pregnancy intentions. Indeed, HIV-positive women in Zambia who knew of PMTCT drugs and believed they were effective were more likely to desire children than HIV-positive women without such knowledge. ${ }^{17}$ Taken together, these findings suggest that previous analyses may not have adequately adjusted for the salient effect of prevalent pregnancy in and of itself on enrollment in care, ART initiation, and incident pregnancy.

In our study, the crude cumulative pregnancy incidence at 1 year was $4.0 \%$ during the pre-ART period and $3.9 \%$ during the on-ART period. Although not directly comparable, the cumulative incidence 1 year after enrollment in the pre-ART period seems lower than the incidence rate reported by Myer et $\mathrm{al}^{10}$ from a multi-country study (6.5 per $100 \mathrm{WY}$ ), and substantially lower than that reported from a single site in Kampala, Uganda when pregnancy tests were used (13.1 per $100 \mathrm{WY}){ }^{6}$ Among patients on ART, more data are available for comparison and suggest that the cumulative incidence of $3.9 \%$ at 1 year after ART initiation observed in our study is lower than the incidence rates reported in other studies from Eastern and Southern Africa, which range from 5.2 to 9.4 per $100 \mathrm{WY}$ in the absence of pregnancy testing, ${ }^{8,10,18}$ and is much lower than that reported when pregnancy testing was used (21.6-24.5 per 100 WY). ${ }^{6,9}$ Of note, however, incidence rates reported in other studies are subject to potentially significant upward bias as they do not take into consideration the competing events of death and LTFU. Pregnancy incidence rates calculated with our data for the pre-ART and on-ART periods (8.6 and 6.7 pregnancies per $100 \mathrm{WY}$, respectively; data not shown) were substantially higher than the cumulative pregnancy incidence that we focus on in this analysis.

With regards to site-level determinants, relative to the AMPATH Consortium, pregnancy incidence was lower among patients at the Infectious Diseases Institute, higher among MTCT-Plus sites, and comparable at FACES sites. This likely reflects differences in program models. The MTCT-Plus Initiative specifically targeted pregnant or recently postpartum women, whereas the Infectious Diseases Institute is a referral center that does not specifically follow pregnant women, and FACES is a routine clinical care program similar to AMPATH. Surprisingly, neither the availability of family planning services within the HIV clinic nor ready access to PMTCT services within the health facility was associated with incident pregnancy. This may reflect that incident pregnancy is largely influenced by individual characteristics and behaviors, but it is also possible that the level of service integration was insufficient to influence women's reproductive behaviors and outcomes. Indeed, a 2013 review of studies examining integration of family planning and HIV services reported weak implementation of the intended integration model in the studies from "realworld" settings, ${ }^{19}$ and a recent study conducted at one of the facilities included in our analysis showed that unmet need for family planning remained high even when contraceptives were provided in the HIV clinic. $^{20}$

This study has several important strengths. With data on nearly 50,000 women with more than 5200 incident pregnancies in $26 \mathrm{HIV}$ clinics in 2 countries with substantial HIV epidemics, it is among the largest assessments of the impact of ART initiation on pregnancy incidence. Additionally, we used several analytic approaches to assess the impact of ART initiation on the likelihood of incident pregnancy, including accounting for longitudinal confounding between factors that are associated with the likelihood of initiating ART and are affected themselves by exposure to ART, and examining potential effect modification by pregnancy status at enrollment in HIV care. This multipronged approach provides a more nuanced understanding of the relationship between ART use and pregnancy 
incidence. We also examined site-level factors associated with pregnancy incidence, which have not been examined previously in relation to incident pregnancy but have been found to be important determinants of other patient outcomes in HIV clinics. ${ }^{21-24}$

Several limitations should also be noted. First, we lacked data on pregnancy desires, which was an important predictor of incident pregnancy among women enrolled in HIV care in a previous analysis. ${ }^{6}$ Similarly, marital status and parity, important predictors of fertility in the general population, were unavailable for many patients in our study and could not be included in models examining predictors of incident pregnancy. However, inclusion of pregnancy status at enrollment in HIV care allowed us to account for some potential confounding because of reproductive history. Additionally, site-level factors were assessed in 2009 but were assumed to have applied to the entire period extending from 2001 to 2009. Given the intensive scale-up of HIV services during that period, it is likely that services became more comprehensive and integrated over time. For example, clinics that had integrated PMTCT and HIV care and treatment services by 2009 may not have had PMTCT services available at all on-site in 2001. Such misclassification may have attenuated the true association between site-level factors and incident pregnancy. Furthermore, site-level variables did not assess use or coverage of PMTCT and family planning services but rather their availability.

\section{CONCLUSIONS}

In conclusion, among a large cohort of women enrolling in HIV care in Kenya and Uganda between 2001 and 2009, we found little evidence that ART initiation is associated with an increased risk of pregnancy in the general population of women enrolling in HIV care. The high incidence of pregnancy we observed both before and after ART initiation underscores the critical importance of enhancing access to reproductive health services for all women in HIV care, regardless of ART status. For women who wish to avoid or delay pregnancy, this entails ensuring access to effective contraceptives, whereas for women who desire children, efforts should focus on promptly ART initiation. Additionally, given the sizable number of women who were pregnant at enrollment in our study, and the elevated risk of incident pregnancy in this group, our findings provide further evidence of the importance of scaling up access to lifelong treatment for pregnant women. Future research should examine whether increased access to Option B+ changes patterns of incident pregnancy among women engaged in HIV care.

\section{ACKNOWLEDGMENTS}

The authors are grateful to all patients and staff at the health facilities included in this analysis: the Academic Model Providing Access to Healthcare program (AMPATH), Eldoret, Kenya; the Family AIDS Care and Education Service program (FACES), Kisumu, Kenya;
Infectious Disease Institute (IDI), Kampala, Uganda; and 3 sites in Columbia University's Mother to-Child HIV Transmission-Plus (MTCT-Plus) Initiative (Nyanza Provincial Hospital, Kisumu, Kenya; St. Francis/St. Raphael Hospital, Nsambya, Uganda; and Mulago Hospital, Kampala Uganda).

\section{REFERENCES}

1. Liotta G, Mancinelli S, Nielsen-Saines K, et al. Reduction of maternal mortality with highly active antiretroviral therapy in a large cohort of HIV-infected pregnant women in Malawi and Mozambique. PLoS One. 2013;8:e71653.

2. UNAIDS WU. Global Update on HIV Treatment 2013; Results, Impact and Opportunities. Geneva, Switzerland: WHO; 2013.

3. Murray CJ, Ortblad KF, Guinovart C, et al. Global, regional, and national incidence and mortality for HIV, tuberculosis, and malaria during 19902013: a systematic analysis for the Global Burden of Disease Study 2013. Lancet. 2014;384:1005-1070.

4. Sinunu MA, Schouten EJ, Wadonda-Kabondo N, et al. Evaluating the impact of prevention of mother-to-child transmission of HIV in Malawi through immunization clinic-based surveillance. PLoS One. 2014;9:e100741.

5. Goga AE, Dinh TH, Jackson DJ, et al. First population-level effectiveness evaluation of a national programme to prevent HIV transmission from mother to child, South Africa. J Epidemiol Community Health. 2015;69:240-248

6. Makumbi FE, Nakigozi G, Reynolds SJ, et al. Associations between HIV antiretroviral therapy and the prevalence and incidence of pregnancy in Rakai, Uganda. AIDS Res Treat. 2011;2011:519492.

7. Maier M, Andia I, Emenyonu N, et al. Antiretroviral therapy is associated with increased fertility desire, but not pregnancy or live birth, among $\mathrm{HIV}+$ women in an early HIV treatment program in rural Uganda. AIDS Behav. 2009;13(suppl 1):28-37.

8. Homsy J, Bunnell R, Moore D, et al. Reproductive intentions and outcomes among women on antiretroviral therapy in rural Uganda: a prospective cohort study. PLoS One. 2009;4:e4149.

9. Schwartz SR, Mehta SH, Taha TE, et al. High pregnancy intentions and missed opportunities for patient-provider communication about fertility in a South African cohort of HIV-positive women on antiretroviral therapy. AIDS Behav. 2012;16:69-78.

10. Myer L, Carter RJ, Katyal M, et al. Impact of antiretroviral therapy on incidence of pregnancy among HIV-infected women in Sub-Saharan Africa: a cohort study. Plos Med. 2010;7:e1000229.

11. Duda SN, Farr AM, Lindegren ML, et al. Characteristics and comprehensiveness of adult HIV care and treatment programmes in Asia-Pacific, sub-Saharan Africa and the Americas: results of a site assessment conducted by the International epidemiologic Databases to Evaluate AIDS (IeDEA) Collaboration. J Int AIDS Soc. 2014;17:19045.

12. Aalen $\mathrm{O}$, Johansen $\mathrm{S}$. An empirical transition matrix for nonhomogeneous Markov chains based on censored observations. Scand J Stat. 1978;5:141-150.

13. Putter H, Fiocco M, Geskus RB. Tutorial in biostatistics: competing risks and multi-state models. Stat Med. 2007;26:2389-2430.

14. Bakoyannis G, Touloumi G. Practical methods for competing risks data: a review. Stat Methods Med Res. 2012;21:257-272.

15. Cole SR, Hernan MA. Constructing inverse probability weights for marginal structural models. Am J Epidemiol. 2008;168:656-664.

16. Kalbfleisch JD, Prentice RL. The Statistical Analysis of Failure Time Data. 2nd ed New York, NY: John Wiley and Sons; 2002.

17. Bankole A, Biddlecom AE, Dzekedzeke K, et al. Does knowledge about antiretroviral therapy and mother-to-child transmission affect the relationships between HIV status and fertility preferences and contraceptive use? New evidence from Nigeria and Zambia. J Biosoc Sci. 2014;46: $580-599$.

18. Andia I, Kaida A, Maier M, et al. Highly active antiretroviral therapy and increased use of contraceptives among HIV-positive women during expanding access to antiretroviral therapy in Mbarara, Uganda. Am J Public Health. 2009;99:340-347. 
19. Wilcher R, Hoke T, Adamchak SE, et al. Integration of family planning into HIV services: a synthesis of recent evidence. AIDS 2013;27(suppl 1):S65-S75.

20. Wanyenze RK, Matovu J, Kamya MR, et al. Fertility desires and unmet need for family planning among HIV infected individuals in two HIV clinics with differing models of family planning service delivery. $B M C$ Womens Health. 2015;15:5.

21. Elul B, Basinga P, Nuwagaba-Biribonwoha H, et al. High levels of adherence and viral suppression in a nationally representative sample of HIV-infected adults on antiretroviral therapy for 6, 12 and 18 months in Rwanda. PLoS One. 2013;8:e53586.
22. Lamb MR, Fayorsey R, Nuwagaba-Biribonwoha H, et al. High attrition before and after ART initiation among youth (15-24 years of age) enrolled in HIV care. AIDS. 2014;28:559-568.

23. Nash D, Wu Y, Elul B, Hoos D, El Sadr W; International Center for AC, et al. Program-level and contextual-level determinants of low-median CD4+ cell count in cohorts of persons initiating ART in eight subSaharan African countries. AIDS. 2011;25:1523-1533.

24. Hoffman S, Wu Y, Lahuerta $\mathrm{M}$, et al. Advanced disease at enrollment in HIV care in four sub-Saharan African countries: change from 2006 to 2011 and multilevel predictors in 2011. AIDS. 2014;28:2429-2438. 